\title{
Médiévales
}

Langues, Textes, Histoire

58 | printemps 2010

Humanisme et découvertes géographiques

\section{L'écho des faits. Quelques remarques sur la prise en compte de la réalité sensible dans des textes optiques, astronomiques et musicaux du début du XIV ${ }^{\mathrm{e}}$ siècle}

The echo of events. Some remarks on the taking into account of perceived reality in the optical, astronomical and musical texts from the beginning of the fourteenth century

\section{Matthieu Husson}

\section{(2) OpenEdition}

\section{Journals}

Édition électronique

URL : https://journals.openedition.org/medievales/5993

DOI : $10.4000 /$ medievales. 5993

ISSN : $1777-5892$

Éditeur

Presses universitaires de Vincennes

\section{Édition imprimée}

Date de publication : 30 juin 2010

Pagination : 113-128

ISBN : 978-2-84292-260-3

ISSN : 0751-2708

Référence électronique

Matthieu Husson, «L'écho des faits. Quelques remarques sur la prise en compte de la réalité sensible dans des textes optiques, astronomiques et musicaux du début du xive siècle », Médiévales [En ligne], 58 | printemps 2010, mis en ligne le 20 septembre 2012, consulté le 24 avril 2022. URL : http:// journals.openedition.org/medievales/5993; DOI : https://doi.org/10.4000/medievales.5993 
Médiévales 58, printemps 2010, p. 113-128

Matthieu HUSSON

\section{L'ÉCHO DES FAITS. \\ QUELQUES REMARQUES SUR LA PRISE EN COMPTE \\ DE LA RÉALITÉ SENSIBLE DANS DES TEXTES OPTIQUES, ASTRONOMIQUES ET MUSICAUX DU DÉBUT DU XIV SIÈCLE}

Depuis que Pierre Duhem a mis à l'honneur des «précurseurs » médiévaux de Galilée, les études sur les différentes formes de prise en compte de la réalité sensible développées par les sciences médiévales se sont multipliées. De la monographie sur un auteur particulier jusqu'à la vaste synthèse en passant par des études sur l'instrumentation d'une discipline ou le vocabulaire employé pour décrire les expériences ${ }^{1}$ et observations, ces travaux ont formé l'image de sciences médiévales qui certes ne disposaient pas de méthodes expérimentales stricto sensu mais possédaient toutes une gamme de moyens d'accès à la réalité sensible.

Les quelques remarques que présente cet article s'inscrivent pleinement dans ce cadre historiographique. Elles sont fondées sur l'étude de textes optiques, astronomiques et musicaux du début du quatorzième siècle dont certains sont devenus des classiques du genre comme l'Expositio de Jean de Murs ou le De iride de Dietrich de Freiberg ${ }^{2}$. Ces trois disciplines sont liées au

1. On peut citer respectivement A. C. Crombie, Robert Grosseteste and the Origins of Experimental Science 1100-1700, Oxford, 1953 ; A. C. CROMBIE, Styles of Scientific Thinking in the European Tradition, Duckworth, 1994, vol. 1, p. 313-423 ; E. Poulle, Équatoires et horlogerie planétaire du XIII' au XIV siècle, Genève, 1980 ; D. JACQUART, «L'observation dans les sciences de la nature au Moyen Âge : limites et possibilités », Micrologus, 4, 1996, p. 55-75.

2. Outre l'Expositio (E. Poulle, «Jean de Murs et les tables alphonsines », Archives d'histoire doctrinale et littéraire du Moyen Âge, t. 47, 1980, p. 241-271) et le De iride (M. STURLESE et L. STURLeSE, Tractactus de iride et de radialibus impressionibus, dans DieTrich VON FreiberG, Opera omnia, K. Flash dir., 4 vol., Hambourg, 1977-1984, vol. 4, p. 95-268), notre corpus d'étude comprend : la Notitia artis musicae, le De musica (JeAn De Murs, Écrits sur la musique, C. Meyer trad., Paris, 2001, p. 53-111, 134-193) et les Tabule permanentes (B. Porres et J. CHABAS, « John of Murs Tabule permanentes for finding true zysygies », Journal for the History of Astronomy, vol. 32, 2001, p. 63-72) de Jean de Murs, le De luce et le De coloribus (M. STURLESE et L. Sturlese, op. cit., p. 1-24, 269-288) de Dietrich de Freiberg, l'Ars (Johannes Boen, Ars (musicae), F. A. Gallo éd., Rome, 1972 (Corpus Scriptorum de Musica, vol. 19)) et le De musica 
quadrivium, aux sciences du nombre - l'astronomie et la musique directement, l'optique comme "science intermédiaire » étant généralement subordonnée à la géométrie et à la physique. L'enjeu de chacune de ces trois disciplines est de mettre en relation les mathématiques et les réalités sensibles qu'elles appréhendent. Elles se rassemblent donc par leur approche mathématique de l'expérience sensible. Nous nous attacherons à comparer la manière dont ces trois disciplines utilisent les différents moyens d'accès à la réalité sensible à leur disposition.

Plus précisément, nous examinerons trois types différents d'accès à la réalité sensible : les observations quotidiennes, l'usage de sources livresques, les modèles expérimentaux. Le rôle de l'observation quotidienne dans la formation des universaux est bien décrit par Aristote par exemple dans la Métaphysique $\mathrm{A}, 1^{3}$. De même, la discussion des opinions des prédécesseurs est au fondement de la méthode aporétique qui doit permettre de fonder les principes de chaque domaine de la science. En revanche, la valeur épistémologique des modèles expérimentaux et des dispositifs de mesure n'est pas donnée à l'avance et doit être construite ${ }^{4}$. Cette élaboration de sens est faite par rapport aux deux types précédents d'accès à la réalité sensible. Ainsi, les différences constatées au niveau de l'utilisation de l'observation et des sources livresques se répercutent dans la manière dont peuvent être utilisés les modèles expérimentaux.

\section{L'observation quotidienne}

On trouve dans chacune des trois disciplines des traces d'informations provenant « spontanément » de la réalité sensible au sens où les auteurs n'ont pas eu à constituer des dispositifs particuliers pour obtenir ces informations.

Dans notre corpus, ce sont les textes optiques qui font le plus souvent référence à des faits, sinon de la vie quotidienne, au moins d'observation courante. Ainsi, au beau milieu d'une énumération sur les différents modes de

(W. Frobenius, Johannes Boens und seine Konsonenzlehre, Stuttgart, 1971) de Jean de Boen, enfin les Tabule magne de Jean de Lignières (Paris, BnF, lat. $7281 \mathrm{f}^{\circ} 201 \mathrm{v}^{\circ}-205 \mathrm{v}$; BnF, lat. 10263 $f^{\circ} 70 \mathrm{r}^{\circ}-78 \mathrm{r}^{\circ}$; Erfurt, Universitätsbibliothek, Amplon. $\left.4^{\circ} 366, \mathrm{f}^{\circ} 28 \mathrm{r}^{\circ}-32 \mathrm{v}^{\circ}\right)$. Nous citerons ces ouvrages par leur titre suivi des numéros des pages de l'édition concernée : «Expositio, p. $256 »$.

3. Cf. Aristote, Métaphysique, A, 1, trad. Tricot, Paris, 1970, p. 3-5. Nous montrerons que les textes de notre corpus souscrivent globalement à la thèse présentée par Aristote dans ce passage.

4. Ainsi, A. C. Crombie souligne qu'il n'y a pas de place pour les observations dans la construction d'une théorie : elles peuvent intervenir au début lors de la recherche des principes d'une science ou d'un art, ou une fois que l'art est constitué pour le confirmer ou l'infirmer globalement ou partiellement. Cf. A. C. CRомвIE, Styles of Scientific Thinking, op. cit., vol. 1, p. 313 . 
radiations permettant l'apparition de couleurs radiantes ${ }^{5}$, Dietrich de Freiberg parle de paons, d'araignées et de canards.

On expérimente ce mode dans la queue du paon et du canard, dans les fils des toiles d'araignées et dans plusieurs autres [situations] ${ }^{6}$.

Le fait que les exemples mentionnés proviennent de l'observation quotidienne est assumé par l'auteur. Ainsi, Dietrich de Freiberg se réfère explicitement à l'observation quotidienne lorsqu'il cherche à établir que le jaune est bien l'une des couleurs de l'arc-en-ciel et n'est pas provoqué par une illusion.

Il est manifeste par l'expérience quotidienne que dans les toiles d'araignées qui, étendues en plein air, ont été arrosées de nombreuses gouttes de rosée proches les unes des autres, en un lieu adapté au soleil et à la vue, ladite couleur apparaît de façon très manifeste, à savoir le jaune, parmi les autres couleurs de l'arc-en-ciel, dans leur lieu et ordre, comme dans l'arc-en-ciel, et aussi proche de la vue <qu'on le souhaite> [...]. De même, elle apparaît de façon très manifeste dans les gouttes répandues sur l'herbe ${ }^{7}$.

Les exemples qu'utilise notre auteur sont manifestement choisis pour renvoyer chacun à une observation qu'il a pu lui-même effectuer. On constate aussi dans cet extrait que l'observation de Dietrich est, malgré sa banalité, structurée par deux idées. Il faut montrer que la couleur jaune n'est pas le fruit d'une illusion. Ainsi, il insiste sur le fait que la couleur peut être perçue d'aussi près que l'on veut. Il faut de plus rappeler que l'apparition et l'ordre des couleurs radiantes dépendent de la position relative de la vue, du soleil et de la goutte. Cette structuration particulière de l'observation est liée au contexte dans lequel elle intervient. Les faits d'observation quotidienne ont un rôle réel dans l'argumentation : il ne s'agit pas simplement d'illustrations et d'ornementations. Plus généralement, les impressions radiantes étudiées par Dietrich appartiennent, pour les principales, à ce domaine de l'observation quotidienne. Ainsi, pour partie au moins, le sujet même du travail est constitué par des informations provenant «spontanément » de la réalité sensible.

5. Les couleurs radiantes sont des rayons de lumières colorés soit à l'issue d'un passage à travers un filtre, soit à l'issue d'un passage à travers un prisme.

6. «Experimur hunc modum in pennis pavonum et anatum et filis telae aranearum et aliis pluribus » (De iride, p. 134). Les traductions françaises, sauf lorsque cela est indiqué, sont de notre fait.

7. «Manifestum est enim per cottidianam experientiam, quod in telis aranearum, quae extensae sub divo multis guttulis roridis et ad invicem propinquis infusae sunt in convenienti situ ad solem et ad visum manifestissime apparet dictus color, scilicet citrinus, inter alios colores iridales suo loco et ordine, sicut in iride, et tam propinque ad visum [...]. Idem manifeste apparet in guttis sparsis in herbis » (De iride, p. 146-147). 
Bien que dans une perspective différente, les sujets traités par l'astronomie proviennent eux aussi en partie de ce domaine de l'observation quotidienne. On en trouve par exemple trace dans le prologue des canons des Tabule permanentes de Jean de Murs.

Tout un chacun, quel que soit son sexe, est à tel point attentif à l'harmonie céleste qu'il semble s'intéresser d'une certaine manière aux conjonctions et oppositions des luminaires. Les gens communs ne s'abstiennent pas de commenter l'influence des pleines et des nouvelles lunes sur les corps humains et la disposition de l'air ${ }^{8}$.

Un socle d'observations quotidiennes - l'observation du mouvement du soleil et des étoiles, des pleines et des nouvelles Lunes, de la succession des saisons - est présent dans nos traités astronomiques malgré leur style technique. Plus encore que ces observations quotidiennes, c'est le sens qu'elles ont pour les observateurs qui les impose aux auteurs. Jean de Murs souligne plus le fait que chacun interprète ces événements que le fait qu'ils sont observés. Au-delà de la nécessité de comprendre un ensemble de phénomènes physiques, ce sont bien leurs significations astrologiques ou calendaires qui sont importantes.

Avec la musique, les phénomènes comme leurs significations sont essentiellement liés aux pratiques musicales. Cela est particulièrement manifeste dans le passage suivant extrait de la Musica de Jean de Murs.

Or, je suis très étonné et j'ignore, à moins que vous ne le sachiez, pourquoi dans nos contrées où règne, par delà le monde, la religion universelle des Fidèles, les deux genres mélodiques que sont le chromatique et l'enharmonique ne sont jamais passés dans l'usage et pourquoi, en revanche, le chant ecclésiastique dans sa totalité, celui qu'inventèrent les saints pères, les savants et les hommes de belle intelligence et dignes de mémoire, pourquoi la totalité de la musique qui est mesurée à l'aide de temps bien précis, celle des conduits, des organa, des mélodies $<$ mesurées $>$, des chansons et autres chants, pourquoi le chant des laïcs, hommes et femmes, jeunes et vieux, enfin pourquoi la musique faite sur tous nos instruments obéissent-ils - sous l'influence de je ne sais quel esprit, si ce n'est la volonté divine et l'instinct naturel - au genre diatonique ${ }^{9}$ ?

8. «Omnis utriusque sexus armoniam celestem adeo contemplatur ut iam de coniunctionibus et opposicionibus luminarium quodammodo se intromittere videtur, vulgares enim a conieccionibus disposicionum aeris et corporum humanorum pro novilunia et plenilunia nequeunt abstinere » (Tabule permanentes, p. 67-68).

9. «Sed miror multum et nescio, si vos, quod in partibus nostris, dico, ubi viget religio catholica fidelium in orbe terrarum, nunquam in usum ceciderunt illa duo genera melorum, chromaticum et enarmonicum ; sed in genere diatonico omnis cantus ecclesiasticus, quem invenerunt sancti patres et doctores et omines bonae mentis et dignae memoriae, omnisque cantus mensuratus per tempora certa, ut in conductis, organis, modulis, cantilenis ceterisque modis, omnisque cantus laicorum virorum et mulierum, iuvenum et senum, omnisque cantus cunctorum nostrorum instrumentorum, nescio, quo spiritu nisi divino quodam nutu et spontanea voluntate naturaliter incidit et favetur » (De musica, p. 186-187, trad. C. MEYER). 
Une première conclusion s'impose : les réalités sensibles étudiées par chacune de nos trois disciplines sont bien différentes. Le socle d'observations quotidiennes étudié par Dietrich de Freiberg n'implique l'homme qu'en tant qu'observateur des faits, la musique au contraire le fait intervenir en tant que producteur des phénomènes. L'astronomie semble être dans une position intermédiaire.

Ces socles d'expériences quotidiennes dans les argumentations jouentils un rôle différent suivant les disciplines ? Dans notre corpus astronomique, seul le passage que nous avons cité renvoie à ce type d'informations. Il est utilisé pour introduire l'étude et en justifier l'intérêt. Il n'a pas de rôle précis dans la construction des canons et des tables. En optique et en musique, la présence de ces passages est accompagnée de l'affirmation d'un empirisme aristotélicien clairement exprimé tant par Dietrich de Freiberg que par Jean de Murs. Ainsi, se référant à Aristote, l'auteur du De iride indique par exemple :

On sait aussi que, selon ce même Philosophe, il ne faut jamais s'écarter de ce qui est manifeste pour les sens ${ }^{10}$.

Jean de Murs cependant exprime cet empirisme de manière plus complète dans l'introduction de sa Musica. Il conçoit bien la science comme une découverte progressive de la structure préexistante du cosmos; on trouve en effet dans la troisième proposition de la première version de la Musica le texte suivant, tout empreint de platonisme :

C'est l'auteur du monde physique qui inscrivit ces admirables consonances dans les choses, et non point l'homme. Les consonances, en effet, existaient avant qu'elles n'apparaissent aux hommes ${ }^{11}$.

L'accès à ces structures cachées de la nature se fait par les sens et par l'accumulation d'observations.

Que toute science et tout savoir résultent de connaissances préexistantes ; qu'il n'existe aucune connaissance antérieure à la connaissance par les sens ; que les positions pour ainsi dire définitives reposent sur un grand nombre d'expériences ; que l'expérience des choses sensibles est à l'origine de l'art ${ }^{12}$.

10. «Scimus autem, quod secundum eundem Philosophum a manifestis secundum sensum nequaquam recedendum est » (De iride, p. 146). Cf. ARIstote, Rhetor. II 3, 1380a18-19.

11. «Auctor naturae mirabiles consonantias fecit rebus insitas et non homo. Praeerant enim consonantiae, antequam hominibus apparerent » (De musica, p. 140-141, trad. C. MEYER).

12. «Omnem doctrinam et omen disciplinam ex preexistensi cognitione fieri. Ante cognitionem sensitivam non aliam inveniri. Experientiae multiplici ut in termino status acquiescere. Experientiam circa res sensibiles artem facere »(De musica, p. 136-137, trad. C. MEYER). Jean de Murs paraphrase ici les passages d'Aristote sur l'expérience de la Métaphysique, A. I, ou des Seconds Analytiques, II. 19. 
Les deux premières propositions justifient théoriquement le rôle fondamental de l'observation : toute science vient d'une cognitio, car la science appartient à la sphère de l'entendement. C'est la perception et donc la connaissance sensible qui est la première des connaissances. La troisième proposition montre bien que c'est la similarité d'un grand nombre d'expériences qui permet d'asseoir une conclusion définitive. La différence avec le principe de reproductibilité, bien que subtile, est importante. Elle change complètement le rapport à l'observation. Une science fondée sur la reproductibilité aura tendance à construire des expériences extrêmement minutieuses dans un environnement maîtrisé, tandis qu'une science fondée sur la similarité ne sera pas tentée par une telle maîtrise de l'expérience ${ }^{13}$. Une certaine variabilité est l'une des conditions assurant la validité scientifique d'une opinion. Cette nécessité de prendre en compte un grand nombre d'observations pour établir un fait explique en partie le recours à l'observation quotidienne dans les argumentations. Les passages tirés du De iride, cités en ouverture de ce paragraphe, fonctionnent selon cette rhétorique : c'est par l'accumulation de cas légèrement différents dans lesquels la couleur jaune est perçue distinctement qu'il est possible de réfuter l'opinion selon laquelle le jaune est une illusion.

L'optique, la musique et l'astronomie s'appuient toutes trois sur un socle « d'expériences quotidiennes » permettant de donner consistance à la discipline et de justifier l'existence des discours. Pour les deux premières, cette caractéristique est associée à l'affirmation d'un certain type d'empirisme ; pour l'astronomie, en revanche, l'observation paraît d'emblée plus construite.

\section{L'utilisation des sources livresques}

Les sources livresques fournissent des informations sur la réalité immédiate déjà théorisées et fortement structurées. Ainsi, Jean de Murs dans son Expositio reprend des passages entiers à Guillaume de Saint Cloud ${ }^{14}$. On trouve chez l'auteur du De iride des exemples de ce type d'utilisation des sources autour des trois questions qui le préoccupent. La théorie aristotélicienne des sens est exploitée dans le traité sur la lumière ${ }^{15}$. La question des couleurs radiantes est abordée notamment à partir de celle des couleurs naturelles

13. Les disciplines médiévales n'étaient cependant pas complètement étrangères au principe de reproductibilité exacte d'une expérience. Ainsi, Avicenne propose dans son Canon (II. 1) sept règles pour garantir la reproductibilité d'une expérience pharmacologique. Cependant, elles seront semble-t-il plus souvent utilisées pour souligner la difficulté de l'opération que pour tenter de la réaliser. $C f$. D. JACQUART, loc. cit., p. 60.

14. Guillaume de Saint Cloud était l'un des principaux astronomes parisiens des années 1290. Ses dates précises ne sont pas connues. Nous conservons trois œuvres : un Directorium, le Kalendarium Regine et l'Almanach planetarum. Cf. J. CHABAs et B. R. GoldsteIn, The Alfonsine Tables of Toledo, Dordrecht, 2003, p. 245, 278-280.

15. De luce, p. 12. 
telle qu'Averroès la présente ${ }^{16}$. Enfin, les Météorologiques d'Aristote sont indispensables au traitement de la question des impressions radiantes ${ }^{17}$. En musique enfin, Jean de Murs comme Jean de Boen reprennent directement à Boèce et à travers lui au traité De l'âme d'Aristote leur conception de la production du son ${ }^{18}$. On lit par exemple dans la Notitia:

Trois choses sont indispensables à la production du son : un agent percuteur, un corps sonore frappé et un milieu. Le premier brise l'air rapidement, le second est un corps physiquement sonore, le troisième est de l'air brisé avec énergie. Il n'y a pas de choc sans mouvement : aussi n'y a-t-il pas de son sans mouvement. Le son par conséquent est le morcellement de l'air provoqué par le choc de l'agent percuteur sur le corps percuté. Il est impossible, en effet, de produire un son dès lors qu'il n'y a qu'un seul élément ${ }^{19}$.

L'utilisation des sources livresques ici structure à tel point les informations que les réalités sensibles semblent s'estomper derrière elles. Ces sources, bien qu'utilisées avec distance critique par nos auteurs, sont pourtant globalement considérées comme donnant des informations sur cette réalité. Dietrich de Freiberg, par exemple, utilise les Météorologiques pour connaître la nature physique des formations atmosphériques dans lesquelles se forment les impressions radiantes qu'il étudie. De même, comme chez Boèce, le son est réellement conçu comme un mouvement par Jean de Murs et Jean de Boen. Ce mode d'accès aux réalités sensibles est commun à nos trois disciplines et les déborde largement. Les sources livresques ne sont cependant pas utilisées seulement comme simple écran devant une expérience possible. La musique et l'astronomie étudient des phénomènes qui, pour des raisons différentes, ne se donnent pas entièrement dans l'instant. Ils ont une dimension temporelle voire historique. Les dispositifs de prise d'informations entièrement livresques sont alors indispensables à l'obtention d'informations sur le passé.

Jean de Boen et Jean de Murs sont conscients de la qualité et de la nouveauté du répertoire de l'Ars nova qu'ils contribuent à transmettre. Ils utilisent ce fait au sein même de leurs raisonnements. On peut distinguer différents niveaux d'élaboration dans le contenu de ces arguments. Au niveau

16. De iride, p. 152-153.

17. De iride, p. 265. La transmission du corpus aristotélicien et de ses commentaires arabes est complexe. L'ensemble de ces textes était disponible, suivant les versions, entre la fin du douzième et le milieu du treizième siècle. $C f$. A. de Libera, La Philosophie médiévale, Paris, 1989, p. 9-22.

18. Voir Boèce, De musica (I. 3). Aristote, De anima (II. 8).

19. « Ad generationem soni tria necessario requiruntur: percutiens, percussum et medium percutiendi. Primum frangens aerem celeriter, secundum corpus sonabile naturaliter tertium aer fractus violenter. Ictus non fit sine motu, ergo neque sonus absque motu est. Est igitur fractio aeris ex impulsu percutientis ad percussum. Nam impossibile est, cum sit unum solum, fieri sonum » (Notitia, p. 60-61, trad. C. MEYER). 
le plus simple, ils ne font que constater une différence entre une pratique « ancienne » et une pratique « moderne ».

Ce que les anciens ont inventé dans les cordes a été ordonné selon les touches par ceux qui sont venus ensuite. Il y a vingt touches sur la main : g-ut, a-re, b-mi etc. Les noms contiennent des lettres et des syllabes, les lettres représentent les touches et les syllabes les notes ${ }^{20}$.

À un niveau plus élaboré, Jean de Boen, par exemple, décrit une progression et donne sens à l'évolution temporelle des pratiques musicales. Ces différentes évolutions peuvent être aussi rationalisées en fonction des lieux et des régions :

Selon les diverses époques et régions beaucoup de nouveautés et de choses non encore entendues peuvent apparaître. Comme, par exemple, la prononciation du comma ou de trois semi-tons mineurs ou de nombreux autres semblables qui, bien qu'ils ne soient pas encore entendus grâce aux nouveaux instruments et à l'habileté des voix le seront plus tard. De même, il n'y avait pas avant Pythagore une subtilité dans le chant identique à celle en usage à notre époque, et nous ne faisons pas la même fracture que les Anglais, les Français ou les Lombards dans le chant ${ }^{21}$.

À un dernier niveau enfin, cette évolution est insérée dans une perception cyclique du temps marquée par la doctrine astrologique de la grande année ${ }^{22}$. Cela est particulièrement clair dans le passage suivant :

La musique a été considérablement affinée par la pratique musicale des modernes, et non point seulement par les expédients ou les trouvailles des gens de savoir qui ont étudié cet art. Au demeurant, le commun des

20. «Quod antiquitas in cordis invenit, hoc posterioritas in clavibus disponebat. Sunt autem claves manuales viginti, scilicet g-ut, a-re, b-mi, etc. Quarum nomina litteras per se continent et sillabas, ut littera clavem et sillabe notas respresentem » (Ars, p. 33).

21. «Nam secundum diversitatem temporis et regionum multa nova et inaudita poterunt suboriri, sicut forte pronuntiatio commatis et trium semitoniorum minorum ac multorum similium, que, licet hactenus non audita sunt, forte tractu temporis per nova instrumenta et vocum habilitates posterius audientur, sicut nec ante Pitagoram fuit tanta subtilitas in cantu, quanta hodiernis temporibus est in usu, nec talem nos, qualem Anglici, G<alli >ci vel Lumbardi in cantu facimus fracturam » (JEAN DE BoEN, De musica, p. 45). Le terme «fractures » fait probablement référence ici aux chants dit en «hoquet ». Dans ce type de composition deux voies se partagent une même ligne mélodique selon un découpage très serré.

22. Selon cette doctrine, toutes les planètes étaient, à l'origine des temps, en conjonction dans le signe du Bélier; une grande année est l'intervalle de temps nécessaire pour que cette situation se reproduise à nouveau. $C f$. G. DE Callataÿ, Annus Platonicus. A Study of World Cycles in Greek, Latin and Arabic Sources, Louvain - Paris, 1996 et C. Burnett et K. Yамамото, Abu Mashar On Historical Astrology : «The Book of Religions and Dynasties : On Great Conjunctions », 2 vol., Leyde, 2000. 
mortels, en particulier les jeunes gens et même les femmes, est poussé à cela. Je ne sais par quel hasard, si ce n'est au gré du génie de la nature réglé par la sphère la plus haute, les choses ne cessent de changer, mais une révolution accomplie, elles reviendront sans doute et retrouveront l'état qui était le leur auparavant ${ }^{23}$.

Ces différents niveaux d'élaboration ne s'opposent pas les uns aux autres. Ils relèvent de la même conception générale présentée plus ou moins complètement suivant les besoins.

C'est cependant probablement en astronomie que ce type d'utilisation des sources livresques est le plus manifeste. Elle étudie en effet non seulement des phénomènes qui se déroulent dans le temps, mais dont le sens et la complexité ne se dévoilent que sur la longue durée. Jean de Murs souligne ainsi dans un beau passage de l'Expositio l'importance des sources livresques en astronomie.

Pour les choses dont la connaissance se trouve dans l'expérience qui n'est éprouvée qu'une fois et ne revient pas semblable, on ne peut que se fier aux auteurs qui en ont fait l'expérience selon ce qui est écrit dans leurs livres, dans lesquels les observations invariables des choses reposent comme dans le trésor de la sagesse ${ }^{24}$.

On ne peut imaginer un passage montrant plus clairement l'importance pour les astronomes des sources livresques dans les progrès de leur discipline. Nos deux auteurs n'abordent pourtant pas ces sources sans esprit critique. Ainsi, Jean de Lignières dans le prologue de ses Tabule magne dresse la liste des causes d'erreurs possibles dans les sources anciennes.

La raison pour laquelle les tables des temps anciens sont fausses et défectueuses ne provient pas d'une erreur des philosophes ptoléméens mais plutôt d'une corruption de leurs racines et peut-être aussi de certains mouvements célestes cachés non encore découverts ${ }^{25}$.

23. «Subtiliataque multum est musica per exercitium modernum non solum litteratorum hominum in hac arte studentium auxilio et inventione, sed et vulgus commune et specialiter iuvenes ac etiam mulieres ad hoc moventur, nescio qua forte nisi naturali industria nunc a superiori regulata. Mutantur enim haec continue et illa revoluto forte circulo aliquo redibunt et erunt sicut prius » (De musica, p. 190-191, trad. C. MEYER).

24. «In illis quorum tota scientia jacet in experiencia que semel experta decetero consimilis non redibit credere non possumus expertis actoribus nisi secundum quod in eorum codicibus scriptum est, in quibus observationes rerum invariabiles sicuti in thesauro sapientie reponuntur » (Expositio, p. 261).

25. «Sed quia hiis temporibus in antiquis tabulis defectus et falsitas accidit non ex errore componentium ptolemerum philosophorum, sed potius ex corruptione radicum illarum vel etiam ex latentia alicuius motus celestis forte nondum inventi »(Tabule magne, Erfurt, Universitätibibliothek, Amplon. $\left.4^{\circ} 366, \mathrm{f}^{\circ} 28 \mathrm{r}^{\circ}\right)$. 
La première cause d'erreurs mentionnée est la corruption des racines ${ }^{26}$. Les causes de corruption des racines peuvent être différentes : il peut s'agir d'une simple imprécision initiale ou bien d'une faute survenue lors de la transmission. La seconde cause d'erreur peut être, dans l'esprit de notre astronome parisien, liée à la complexité même du mouvement céleste et au fait que certains mouvements très lents ne peuvent être découverts que grâce à une très longue période d'observation. Jean de Lignières a probablement en tête ici le mouvement de la huitième sphère ${ }^{27}$. Nous savons, particulièrement à travers des textes de Jean de Murs, que ce mouvement préoccupait les astronomes parisiens de cette première moitié du quatorzième siècle. C'est l'existence de ces mouvements cachés qui rend chaque observation potentiellement unique malgré l'aspect cyclique des phénomènes étudiés. Jean de Murs s'interroge lui aussi, autour d'un cas concret, sur les causes possibles d'erreurs dans les sources astronomiques :

Voilà pourquoi je dis que dans nos livres et dans ceux que nous avons vus est écrit $216 ; 3713^{\circ}$ et nous estimons cela erroné par 20 minutes de degré. Il s'agit ou d'une erreur de l'imagination comme cela arrive souvent ou d'une erreur du scribe qui croyant écrire 17 a écrit 37 . Alphonse étant un excellent et patient astronome, on ne peut lui imputer cette erreur, mais on doit raisonnablement l'imputer à un scribe qui a mal copié, et il est possible de trouver des erreurs dans d'autres racines du soleil à d'autres époques, car elles ont leur origine dans cette racine d'Alphonse ${ }^{28}$.

Les sources livresques sont effectivement utilisées par nos auteurs pour obtenir des informations sur la position des astres dans un passé récent ou ancien ${ }^{29}$. Ainsi, tandis que c'était le rôle de l'homme (comme simple observateur ou comme producteur de faits) qui marquait la différence entre les disciplines étudiées dans le domaine de l'observation quotidienne des réalités sensibles, c'est le rapport à la temporalité qui définit chez elles des utilisations différentes des sources livresques. Examinons maintenant le cas des modèles expérimentaux et des dispositifs de mesures.

26. Les racines sont des valeurs indiquant les positions des planètes ou d'autres « objets » célestes à une date fixe du passé. Elles servent de point de départ dans les calculs astronomiques.

27. La huitième sphère ou sphère des étoiles fixes est animée d'un mouvement afin de prendre en compte la précession des équinoxes. Différentes conceptions s'affrontent : celle d'un mouvement continu (Ptolémée), celle d'un mouvement d'accès et de reccès (tables de Tolède), celle d'une combinaison des deux (tables alphonsines). J. ChaBAs et B. R. GoldsteIn, The Alfonsine Tables, op. cit., p. 255-266.

28. «Quod ideo dico quia in libris nostris et eis quos vidimus scriptum est 2.16;37.13 quam de 20 minutis unius gradus estimamus corruptam vel ex ymaginatione ut pluries accidit aut ex vicio scriptoris mutatam ut, credens scribere 17 , scripsit 37 ; ideoque Alfonsio tam excellenti patientissimoque astrologo non imponatur hoc vitium sed rationabilius scriptori male exemplanti ; et inde corruptionem contingit reperiri in aliis radicibus solis aliorum temporum propter hoc quia ortum habent ex illa radice Alfonsii » (Expositio, p. 264).

29. Cf. Expositio, p. 254, p. 264 etc. 


\section{Modèles expérimentaux et dispositifs de mesures}

Les dispositifs de prise d'informations que nous abordons maintenant ressemblent, au moins par trois caractéristiques, au type de procédures mis en place par la méthode expérimentale moderne : ils décrivent des « expériences » en milieu contrôlé ; le rôle rhétorique apparent de ces «expériences » est de confirmer ou d'infirmer une « hypothèse »; dans la plupart des cas, ils impliquent sinon une quantification au moins l'utilisation de contenus mathématiques. Ce sont ces éléments qui justifient l'attention particulière que leur portent les historiens des sciences.

Cette attention a cependant conduit, dans bien des cas, à souligner le caractère superficiel de ces similitudes ${ }^{30}$. Il est ainsi tout simplement particulièrement rare que la documentation que nous possédons permette de trancher avec certitude la question de savoir si l'expérience que nous décrit une source a réellement eu lieu. Il est parfois certain que l'expérience n'a pas eu lieu. C'est le cas lorsque l'expérience décrite est matériellement impossible. Certaines descriptions figurées proposées par Dietrich de Freiberg, tout en étant compatibles avec les principes fondamentaux de l'optique géométrique médiévale, sont en contradiction avec ce que l'on connaît aujourd'hui de cette discipline $^{31}$. Plus rarement encore, nous pouvons à l'inverse être sûrs qu'une expérience a réellement été réalisée. L’observation de Guillaume de Saint Cloud à propos de la date de l'équinoxe de printemps ne pouvait être déduite à partir des tables à sa disposition. Nous sommes alors certains qu'il y a bien eu observation. Dans la plupart des cas cependant, nous devons nous contenter d'une présomption.

Ces difficultés sont accentuées par le fait qu'il existe toute une série « d'expériences » qui se transmettent de texte en texte sans avoir besoin d'être reproduites. Un exemple banal existe en théorie musicale : celui de la toupie ; Jean de Murs et Jean de Boen le reprennent tous deux à Boèce ${ }^{32}$.

30. Par exemple, lorsque A. C. Crombie examine les différents styles de pensée scientifique qui ont contribué à la « révolution scientifique » du dix-septième siècle, il considère que l'apport des «philosophes scientifiques » médiévaux est essentiellement épistémologique, tandis que le développement des techniques expérimentales seraient principalement le fait des « artistes de la renaissance » : cf. A. C. Crombie, Science, Optics and Music in Medieval and Early Modern Thought, Londres, 1990, chap 7 et 8, p. 139-175; ou bien note 4 pour une référence plus précise.

31. Comparer par exemple les figures 20 et 37 de l'édition du De iride (p. 440 et 444). Ces deux figures montrent les rayons d'une source lumineuse réfractée par une sphère diaphane. Dans les deux cas, un foyer de convergence est représenté (ce qui est en contradiction avec les lois de l'optique moderne). Il est symétrique de la source lumineuse dans la figure 20 et, en contradiction avec la figure 20 , non symétrique dans la figure 37 . L'éditeur du texte fournit une édition critique des figures présentes dans deux des trois manuscrits contenant le De iride.

32. Voir Boèce, De musica (I. 3) ; JeAn de Murs, Notitia, p. 60-61; JeAn de Boen, De musica, p. 68-69. 
Ces obstacles sont, en partie au moins, le reflet des difficultés rencontrées par les auteurs médiévaux eux-mêmes pour donner sens à ce type de procédés. La manière dont Jean de Murs expose la légende des marteaux de Pythagore ${ }^{33}$ dans la Musica est sur ce point particulièrement éloquente ${ }^{34}$. Trois types d'obstacles sont distingués. Le premier concerne les objets étudiés et la difficulté de contrôler effectivement les différents paramètres de l'expérimentation. Le second est relatif à l'observateur et la fiabilité des organes des sens. Le dernier obstacle est d'ordre épistémologique. Il souligne le saut qui existe entre une perception sensible particulière et une connaissance rationnelle générale.

Au début du quatorzième siècle, l'optique, la musique et l'astronomie héritent pourtant de leur histoire ancienne ou récente certains objets ou situations d'observations qui occupent dès lors une place privilégiée. Ainsi, les observations d'équinoxes, de solstices, d'éclipses et de conjonctions sont, au moins depuis l'Almageste, des exemples paradigmatiques en astronomie. De même, le De musica de Boèce a institué le monocorde comme l'instrument essentiel de la théorie musicale. Enfin, les prismes, les grosses ou petites boules de cristal, peut-être aussi les ballons remplis d'eau, constituent pour Dietrich de Freiberg et, avant lui, pour Albert le Grand ou Roger Bacon, des outils privilégiés d'étude de la formation des couleurs radiantes ${ }^{35}$.

Tous nos auteurs doivent donc construire le sens de ces situations d'observations particulières et chacun le fait d'une manière différente. C'est ainsi dans ce dernier domaine que les contrastes entre nos trois disciplines sont les plus marqués. Quoi de commun entre la musique, qui s'appuie sur un instrument simple proposant des situations d'observations stéréotypées et explorées depuis l'Antiquité, l'optique, qui utilise toute une variété d'objets dont les potentialités commencent juste à être perçues, et l'astronomie, qui doit mettre en place des dispositifs de calculs et mesures lourds et dispose de peu d'opportunités d'observations pertinentes ? À côté des dispositifs expérimentaux, les manières de décrire et de rendre compte des observations dans les textes sont aussi très variées. Ainsi, l'utilisation du monocorde, la mention d'une observation astronomique ancienne ou encore d'une expérience attribuée à Alhacen sur la réfraction du rayon lumineux ne donnent pas lieu à une rhétorique particulière ; en revanche, l'utilisation des prismes ou des «exemples figurés » en optique, la narration de la légende de Pythagore en

33. Selon cette légende Pythagore, se trouvant à proximité d'un atelier de forgeron, reconnut l'octave, la quinte, la quarte et le ton parmi les sons produits par les frappes des marteaux sur les enclumes. Par la suite il pesa les marteaux et put ainsi établir les proportions fondamentales de la science musicale. $C f$. BoÈce, De institutione musica, I, 10, trad. C. Meyer, Turnhout, 2004, p. 47-51.

34. De musica, p. 138-139.

35. Voir De iride, XIV-XV, XXX-XXXV. 
musique ou la description d'une observation par son propre auteur en astronomie induisent une véritable créativité lexicale ou littéraire. Enfin, c'est naturellement l'examen attentif du statut épistémologique et des différents rôles que tiennent ces observations privilégiées dans les argumentations qui constitue le principal champ de recherche sur ces questions. Parmi ces différentes possibilités, les brèves remarques finales de ce travail porteront sur les différentes manières dont le problème du saut épistémologique entre une observation particulière et une connaissance générale est abordé. On trouve ainsi dans l'Expositio le passage suivant :

Ce qui vient d'être dit de la longueur vraie de l'année peut être éprouvé par deux observations du lieu du soleil entre lesquelles se trouvent 134 années solaires et on verra ainsi si notre discours est conforme à la vérité des choses. ${ }^{36}$

Il est évident qu'un homme seul ne peut lui-même effectuer le programme d'observations proposé par Jean de Murs. Il convient donc à celui qui souhaite vérifier que les raisonnements proposés sont conformes à la «vérité des choses » de se reposer sur le témoignage d'autrui. Celui-ci peut lui être transmis sous différentes formes: soit sous la forme simple d'une observation de la position de l'astre, soit sous celle plus complexe d'une table permettant de calculer cette position. Ainsi, une « observation » peut désigner aussi bien une mesure concrète de la position d'un astre à un instant donné, qu'un calcul effectué au moyen de tables et, plus souvent encore, un mélange, souvent difficile à démêler, de ces deux procédés ${ }^{37}$. Ces ambiguïtés sont utilisées en permanence dans l'Expositio pour éprouver la cohérence du modèle tabulaire des mouvements célestes et pour le confronter à des mesures. Ainsi, en astronomie, le saut épistémologique du particulier au général se fait par une mise en relation fine, au moyen d'outils mathématiques, entre les informations obtenues par le biais de mesures et celles obtenues par les sources livresques.

Lorsque, dans le De iride, Dietrich de Freiberg aborde le problème de la formation des couleurs radiantes, il annonce pour la première fois qu'il va se servir d'un prisme hexagonal. On s'attendrait, à la suite de cette annonce, à une exposition d'observations expérimentales impliquant le prisme. Nous trouvons à la place la description d'une figure géométrique représentant un prisme et un rayon lumineux ${ }^{38}$. En fait, les observations impliquant l'utilisation d'un objet

36. «Quod autem dictum est nunc de vera quantitate anni potest experiri per duas observationes de loco solis inter quas cadunt anni Christi solares 134, et tunc videbitur si sermonibus consonet veritas rei » (Expositio, p. 253).

37. La polysémie du mot en astronomie est reconnue depuis longtemps par les spécialistes. Voir par exemple E. Poulle, « L'instrumentation astronomique médiévale », dans B. RiBÉMONT éd., Observer, lire, écrire le ciel au Moyen Âge, Paris, 1991, p. 254.

38. De iride, p. 158. 
particulier et une implication forte de l'observateur dans les textes optiques du dominicain sont, pour la plupart, présentées sous cette forme d'une figure géométrique et de sa description dans le texte. Elles sont souvent exposées en série $^{39}$ et insérées au sein d'argumentations présentant aussi des observations quotidiennes ${ }^{40}$. Une des principales différences entre ces «expériences » et les observations quotidiennes est le fait que les premières sont décrites à l'aide de figures géométriques. Ce procédé n'est pas anodin. La figure en effet est un peu plus qu'une observation. Elle est construite à partir des règles universelles de l'optique et on peut déduire de l'observation d'une seule des règles qui, elles aussi, seront universelles ${ }^{41}$. Ainsi, pour Dietrich de Freiberg, c'est l'ambiguité qui existe entre une expérience et sa représentation figurée qui est utilisée pour opérer le saut du particulier au général. Au lieu d'un dialogue avec les sources livresques comme en astronomie, les expériences prennent sens par rapport aux observations quotidiennes. Les situations de l'optique et de l'astronomie sont ainsi, sans être identiques, assez similaires dans leur façon d'utiliser les mathématiques comme instrument de recherche ${ }^{42}$.

La musique propose des solutions différentes. Jean de Murs structure la narration de la légende de Pythagore de manière à la rendre conforme aux règles de l'épistémologie aristotélicienne. Il montre de quelle manière Pythagore forme, à partir de différentes expériences sensibles, un universel apte à servir de principe à la science musicale ${ }^{43}$. Dans le De musica de Jean de Murs, ce rôle fondamental de la légende de Pythagore est souligné par le fait qu'à partir de son énoncé le texte adopte une structure déductive. Pour Jean de Murs, la « découverte » de Pythagore porte sur la nature physique du son et s'exprime de manière mathématique au moyen des différentes proportions formées entre les nombres 6, 8, 9 et 12. Jean de Boen place la narration de la légende de Pythagore à la fin de son Ars et l'utilise ainsi pour marquer la frontière entre le chantre et le musicus. Il semble apparemment souscrire, à sa manière de chantre, au même point de vue que Jean de Murs. Il exprime, au moyen des mêmes nombres, les résultats de l'expérience de Pythagore. Pour lui cependant,

39. Voir par exemple les chapitres traitant du lieu des couleurs radiantes De iride, p. 168-184.

40. Voir le passage sur la couleur jaune dans l'arc-en-ciel De iride, p. 145-148.

41. Pour un examen précis de la manière dont Dietrich de Freiberg utilise les figures dans ses textes optiques voir M. Husson, «Les figures dans les textes optiques de Dietrich de Freiberg », dans J. Biard, D. Calma et R. Imbach, éd., Recherches sur Dietrich de Freiberg, Turnhout, 2009, p. 239-264. Pour une référence plus générale sur le rôle des figures au Moyen Âge voir : Testo $e$ immagine nell'alto medioevo, 2 vol., Spolète, 1994 (Settimane di studio del Centro italiano di studi sull'alto medioevo, 41 ).

42. Cette similarité pourrait être, entre autres, la conséquence du fait que l'optique arabe a modelé son usage des expériences sur le modèle de l'astronomie : voir A. I. SABRA, « The astronomical Origin of Ibn al-Haytham's Concept of Experiment », dans Actes du Congrès international d'histoire des sciences (Paris 1968), Paris 1971, t. III A, 133-136.

43. Notitia, p. 66-67 et le De Musica, p. 138-139. 
ces proportions ne reposent pas sur la physique du son mais reflètent les goûts fondamentaux de l'âme humaine. Il s'appuie alors sur une autre série d'autorités pour soutenir sa thèse ${ }^{44}$.

Ainsi, en musique comme en astronomie et en optique, la généralité s'exprime de manière mathématique et les expériences prennent sens dans un dialogue avec les autres sources d'informations sur les réalités sensibles. La musique étant, comme l'astronomie, une discipline marquée par la temporalité, c'est naturellement par rapport aux sources livresques que les expériences font sens. Cependant, le procédé par lequel le saut du particulier au général est réalisé en musique est très différent de ceux employés en optique et en astronomie. Pour ces deux dernières, les mathématiques ne sont pas simplement le lieu d'expression de la généralité, mais bien plus encore l'outil qui permet de tisser un lien intime entre les «expériences » et les autres sources d'informations sur la réalité par rapport auxquelles elles prennent sens. En optique et en astronomie, les mathématiques et les expériences sont un outil d'exploration. En musique, c'est une narration qui tisse les liens permettant de donner sens à l'expérience et, lorsque Jean de Boen met en question les principes qui résultent de cette narration, il le fait sur des bases esthétiques et psychologiques qui resteront pour longtemps encore hors de portée de toute méthode expérimentale.

Matthieu Husson - Paris, équipe EA 4116 «Savoirs et pratiques du Moyen Âge à l'époque moderne » (EPHE), hussonmatthieu@gmail.com

\section{L'écho des faits. Quelques remarques sur la prise en compte de la réalité sensible dans des textes optiques, astronomiques et musicaux du début du XIV ${ }^{\mathrm{e}}$ siècle}

Les études sur les différentes formes d'empirisme développées par les sciences médiévales sont nombreuses. De la monographie sur un auteur particulier jusqu'à la vaste synthèse, en passant par des études sur l'instrumentation d'une discipline ou le vocabulaire employé pour décrire les expériences et observations, ces travaux ont formé l'image de sciences médiévales qui certes ne disposaient pas de méthodes expérimentales stricto sensu mais possédaient toute une gamme de moyens d'accès à la réalité sensible allant, dans un vocabulaire moderne, de la simple observation quotidienne jusqu'à la mise en place de dispositifs complexes de mesures.

44. Jean de Boen associe alors une comparaison entre la vue et l'ouïe issue de la tradition aristotélicienne (Des sens et du sensible Chap. 5 et De l'âme II. 7 et 8) à une référence à certains arguments attribués à Nicomaque par Jérôme de Moravie dans son Tractatus de musica (Hieronymus de Moravia, Tractatus de musica, S. M. Cserba éd., Münster, 1935, p. 64). 
Les quelques remarques que présente cet article s'inscrivent pleinement dans ce cadre historiographique. Elles sont fondées sur l'étude de textes optiques, astronomiques et musicaux du début du quatorzième siècle dont certains sont devenus des classiques du genre comme l'Expositio de Jean de Murs ou le De iride de Thierry de Freiberg. Nous souhaitons mettre en lumière ici certaines différences entre ces trois disciplines. Le type de « réalité » qu'elles ont à étudier est bien différent. Ces spécificités apparaissent directement aux auteurs médiévaux par le biais de l'observation quotidienne, mais elles induisent aussi des contrastes importants au niveau des autres moyens d'accès à la réalité sensible.

expérience - quatorzième siècle - optique - musique - astronomie

\section{The echo of the events. Some remarks on the taking into account of perceived reality in the optical, astronomical and musical texts from the beginning of the fourteenth century}

There are numerous studies of the different forms of empiricism developed by mediaeval science. From monographs devoted to a particular author up to vast syntheses, passing through studies of some specific instrument of a particular discipline or the vocabulary employed to describe experiments and observations, these studies have formed an image of mediaeval science that certainly did not have experimental methods at its disposition but that possessed a whole range of methods capable of perceiving reality going, in a modern vocabulary, from simple daily observations to the establishment of more complex systems of measurement. The remarks presented in this article are fully situated in a historic perspective. They are based on the study of optical, astronomical and musical texts from the beginning of the 14th century of which some have become the classics of the genre such as the Exposito of Jean de Murs or De Iride of Thierry de Freiberg. We wish to cast light on certain differences among these three disciplines. The type of "reality" that they study is quite different. These specificities are directly apparent to the mediaeval authors through daily observation but they also induce important contrasts at the level of other methods of perceiving reality.

experiment - fourteenth century - optics - music - astronomy 